\title{
La educación para el siglo XXI: una perspectiva holística del aprendizaje
}

Karen María Acevedo Mena ${ }^{1}$

\section{Información}

\section{de artículo:}

Recibido: 30/09/2019

Aprobado:30/10/2019

\section{Palabras claves:}

Contenidos,

Docente,

Educando,

Enseñanza-Aprendizaje,

Perspectiva Holística.

\section{Keywords:}

Contents,

Teaching,

Educating,

Teaching-Learning,

Holistic Perspective.

\section{Resumen}

El presente escrito está basado en una reflexión sobre cómo debemos de concebir la educación para el siglo XXI, en el cual se plantea que la educación debe abordarse desde una perspectiva holística del aprendizaje, puesto que los cambios sociales y culturales que se han producido en los últimos tiempos nos demanda que modifiquemos la visión que se tenía de la educación en el siglo pasado. Los pedagogos hablan entonces, de una acción educativa integral para abarcar la totalidad del ser, en donde prevalezcan las necesidades individuales (cognitivoafectivas) de los educandos. En el desarrollo del planteamiento se enfatiza en cómo aplicar el concepto del holismo en el proceso de enseñanza-aprendizaje, en el rol del educando, en el rol del docente y en los contenidos. Por último, se destaca la importancia del compromiso que debemos adquirir como educadores para responder a las exigencias que demanda nuestra sociedad en relación con la educación.

\section{Education for the 21 st century: a holistic perspective of learning}

\begin{abstract}
This paper is based on a reflection on how we should conceive education for the 21st century, in which it is stated that education should be approached from a holistic perspective of learning, since the social and cultural changes that have occurred in recent times, it demands that we modify the vision of education in the last century. The pedagogues then speak of an integral educational action to encompass the totality of being, where the individual (cognitive-affective) needs of the students prevail. The development of the approach emphasizes how to apply the concept of holism in the teaching-learning process, in the role of the

${ }^{1}$ Máster en Psicología Clínica y de la Salud, Profesora e la Universidad Nacional Autónoma de Nicaragua-Managua (UNANMANAGUA). E-mail: karenacevedomena@hotmail.com, (1): https://orcid.org/oooo-ooo2-3449-260o
\end{abstract}


student, in the role of the teacher and in the contents. Finally, the importance of the commitment that we must acquire as educators to respond to the demands that our society demands in relation to education is highlighted.

\section{Introducción}

En los últimos tiempos se han producido cambios sociales y culturales significativos que hacen imprescindible cuestionarnos cómo debe ser la educación para el siglo XXI. Puesto que la sociedad avanza a pasos gigantescos y la educación debe avanzar con ella.

En el campo de la educación cada día más profesores comprenden que las influencias educativas no operan literalmente ni de manera igual para todos los sujetos y que por tanto no pueden ser estandarizadas (Fernández, 1993). A su vez, Cabrera (2009) señala que la personalización de la educación se perfila ya como uno de los principales objetivos y retos de los sistemas educativos contemporáneos. Estos planteamientos nos llevan a inferir que la educación debe considerar en sus principios atender a la diversidad, y al mismo tiempo debe ser integral. Esta integralidad sólo puede lograrse si se posee una concepción holística de un fenómeno tan complejo como lo es la educación.

Por tanto, el presente escrito plantea que la educación para el siglo XXI debe abordarse desde una perspectiva holística del aprendizaje. Antes de justificar este planteamiento, primero definiremos el significado de educación y su relación con los aspectos culturales de una sociedad. León (2007) define la educación como un proceso humano y cultural complejo en el que se debe tomar en cuenta la condición y naturaleza del hombre y de la cultura en su conjunto, además lo particular del individuo tiene sentido por su vinculación e interdependencia con los demás y con el conjunto.

Este autor también refiere que "el hombre llega a tener la imagen de la cultura a través de la educación y el aprendizaje" (León, 2007, p.598). Esta afirmación resfuerza nuestro esbozo sobre la importancia de concebir la educación desde una visión holística del aprendizaje puesto que en este sentido se considera que educar es formar sujetos y no objetos, cuyo propósito es completar la condición humana del hombre, adaptarlo al medio tomando en cuenta sus características individuales, biológicas, cognitivas, psicológicas y culturales, enfatizando en la promoción de valores como la honestidad, la ética, la justicia, la equidad, la disciplina, la prudencia, la compasión, la empatía y convirtiendo al hombre en un sujeto autónomo que gane su propio pan, su propia vida, que construya su propio proyecto y descubra su lugar en el mundo.

Basándonos en estas primeras consideraciones, en seguida se abordará en qué consiste el aprendizaje holístico, caracterizando cuál es el rol que debe desempeñar el educando, el docente y los contenidos desde esta perspectiva. 


\section{La educación y el aprendizaje basado en el concepto del holismo}

Conviene mencionar que se expondrá de manera comprensible cuáles son los fundamentos para aplicar una teoría holística de la educación. Santos Rego (200o) describe que los educadores nos vemos desbordados por tanta movilidad y diversidad, por tanta información que existe hoy en día con el uso del Internet y las TICS por lo que el proceso educativo se vuelve complejo. Los pedagogos hablan entonces, de una acción educativa integral para abarcar la totalidad del ser. Este autor menciona que el "término "holístico" opera semánticamente sobre el lexema "holos", elemento compositivo que interviene antepuesto en la formación de muchas palabras, primero latinas y luego romances, con un significado de totalidad" (p.135).

De esta manera intentar una comprensión holística de la realidad es favorecer un análisis estructural no fragmentario de modo tal que el todo es siempre más, mucho más que la suma de las partes. Este enunciado científico nos recuerda los referentes teóricos para comprender y representar los procesos educativos (Santos Rego, 2000).

La comprensión holística también es de gran utilidad puesto que es imposible reducir los problemas educativos a cualquiera de sus variables o dimensiones. La realidad no se puede comprender de manera unidimensional, se necesita tener la visión de que existen un sin número de variables interrelacionadas que inciden en la educación específicamente en el proceso de enseñanza-aprendizaje.

Si se logra esta visión, el autor señala que la escuela favorecería a la inclusión y no a la exclusión dentro de sus recintos, además se respetaría la diversidad cultural aprovechando sus ventajas para fortalecer un sentido de comunidad y de civilidad. Una de las esencias de la educación en el holismo se basa en educar al ser humano como un todo.

En términos de Rinke (1982) citado por Santos Rego (2000) la educación holística es un "modelo funcional, integrado y generalizado de educación que se centra en toda la situación de enseñanza-aprendizaje y que varía la estrategia sobre ésta para tomar en cuenta las necesidades del alumno, del profesor, y de la misma situación" (p.141).

Es decir en la educación holística prevalece las necesidades individuales (cognitivo-afectivas) de cada educando por lo que el docente debe diseñar estrategias de enseñanza-aprendizaje que respondan a estas necesidades para lograr un mejor aprovechamiento académico. En esto, juega un papel fundamental la motivación ya que beneficia a gran escala el aprendizaje del individuo.

La enseñanza y el aprendizaje deben verse como parte de un único proceso que tiene como fin la formación del educando (Espinoza, 2017). Así mismo: 


\section{REFLEXIÓN EDUCATIVA}

Álvarez de Zayas (1988), expone que una posición teórica y práctica importante es considerar y concebir el proceso de enseñanza-aprendizaje de forma bilateral; es decir, está conformado tanto por la actividad de enseñar, como la de aprender, y es así que se reconoce en la literatura: como un proceso que se caracteriza por la unidad dialéctica entre el profesor y los estudiantes (Espinoza, 2017, p.8).

Esta visión es integral, por lo tanto constituye una perspectiva holística del aprendizaje pero ¿qué es el aprendizaje? Monereo (1990) citado por Espinoza (2017) conceptualiza el proceso de aprendizaje como una actividad individual que se desarrolla en un contexto social y cultural. Es resultado de procesos cognitivos individuales mediante los cuales se asimilan e interiorizan informaciones, se construyen nuevas representaciones mentales significativas y funcionales para después aplicarse a situaciones diferentes a los contextos donde se aprendieron.

Vigotsky (1995) citado por Cabrera (2013) define el aprendizaje como:

Un proceso que posee tanto un carácter cognitivo como socio-afectivo, y que por tanto implica la personalidad como un todo, propiciando que el sujeto se apropie de la cultura desarrollada por la sociedad, mediante su actividad y con la ayuda de los otros, por medio de los instrumentos y sistemas de signos construidos históricamente por la humanidad (p.167).

Este autor también menciona que en muchos casos se asume una visión atomizada sobre el aprendizaje, entendiéndolo básicamente como un proceso asociado a la percepción y al procesamiento de la información, que enfatiza lo cognitivo, lo intelectual, lo informativo sobre lo afectivo-emocional, lo vivencial. Sin embargo el autor considera que se debe asimilar el aprendizaje desde un enfoque holístico ya que nos permite apreciar el carácter individual de este proceso, expresado en un estilo personal del sujeto al aprender y en el cual se refleja la personalidad vista como una unidad de sus componentes cognitivos y afectivos.

En ambas definiciones se evidencia que en el proceso de aprendizaje no sólo se deben tomar en cuenta los aspectos cognitivos del individuo sino también los aspectos positivos, sociales y culturales, lo que reafirma lo expuesto anteriormente por León (2007).

\section{Rol del educando}

La educación puede y debe ser pensada como un todo (o totalidad), por consiguiente se debe considerar los procesos sociales al mismo tiempo que los lógicos y culturales (Maldonado, 2014). Este enunciado enfatiza la aplicación del concepto del holismo en la educación. 
Aquí continuaré refiriendo lo expuesto por Maldonado (2014) al señalar que los jóvenes en las universidades pertenecen a nuevos contextos culturales muy distintos a los de la gran mayoría de sus profesores. El uso de la tecnología es el factor principal que está generando cambios culturales transcendentales, dentro de los cuales se encuentran las dinámicas y procesos educativos (enseñanza-aprendizaje).

Dentro de estas circunstancias emerge la necesidad de comprender la educación como un fenómeno dinámico y no estático, que se caracteriza en el mundo contemporáneo por entornos cambiantes. La perspectiva holística del aprendizaje aporta a lograr esta visión de una manera más clara.

Precisamente por estas razones el educando se sitúa como un sujeto activo dentro del proceso de enseñanza-aprendizaje. Para ampliar este rol del educando haré referencia a los aportes generados por el enfoque constructivista el cual según Chadwick (2006):

El planteamiento de base de éste consiste en que el individuo es una construcción propia que se va produciendo como resultado de la interacción de sus disposiciones internas y su medio ambiente, y por lo tanto, su conocimiento no es una copia de la realidad, sino una construcción que hace la persona misma (p.112).

El autor también señala que entonces el aprendizaje no es un asunto de transmisión y acumulación de conocimientos sino un proceso activo por parte del educando que consiste en enlazar, extender, restaurar e interpretar, es decir, construir conocimiento desde su experiencia y la información que recibe. Esto constituye la base para que el aprendizaje sea significativo para el educando.

\section{Rol del docente}

El primer desafío que debe asimilar el docente es comprender que el educando es un ser humano biopsicosocial y que vive en un contexto cultural cambiante.

Es por ello que las aulas de clase, seminario, taller o laboratorio pueden y deben ser vistos por los docentes como sistemas abiertos sensibles a los procesos y dinámicas del entorno. En este punto es importante aludir que en el proceso de enseñanzaaprendizaje ya no debe existir centralidad ni una jerarquía rígida sino ver este proceso como multinivel, en paralelo o también horizontal (Maldonado, 2014).

Cabe mencionar que este autor contrasta la forma como se visualiza el futuro en el proceso de enseñanza-aprendizaje. De un lado, la educación tradicional que lamentablemente sigue predominando en muchos lugares, consiste en la utilización de pizarras, papelógrafos, aula unifuncional, centralidad del profesor, linealidad y jerarquía. En contraste está lo que en general puede señalarse como la nueva aula, 
la cual se desarrolla alrededor de: pizarra interactiva, tablet y toda clase de nuevas tecnologías de aprendizaje. Es decir se caracteriza por horizontalidad, intercularidad y no linealidad. La no linealidad puede ser entendida como el trabajo para resolver problemas y estos tienen más de una solución posible. En este sentido, el docente no puede sentirse el amo y dueño del conocimiento o el único que tiene la solución correcta.

Delval (2013) plantea que el profesor es una pieza central en el funcionamiento del proceso educativo y mientras no cambie la función de los profesores, no habrá ningún cambio ni será posible ninguna reforma educativa. Puesto que todas las reformas educativas fracasan porque se hacen leyes, se hacen reglamentos pero parece olvidarse que son los profesores los que tienen que administrar todo eso y si los profesores continúan con sus mismas prácticas no se producirá los cambios necesarios. También el profesor debe crear situaciones de aprendizaje, impulsar la realizaciones de esas actividades, ponerlas en práctica e incentivar a los educandos a que las desarrollen orientándolos en las dificultades y promoviendo la autonomía y la responsabilidad del educando.

Por consiguiente, el docente desempeña un rol de facilitador, quien ayuda al individuo o grupo a entender los objetivos comunes y contribuye un plan para alcanzarlos. Silva (2010) menciona que el docente pasa de ser un transmisor de conocimientos a facilitador del aprendizaje, promoviendo y orientando por medio de la construcción del producto, resultado individual y de la interacción social.

Dentro de este rol también se evidencia la necesidad de comprender la educación para el siglo XXI con una visión holística del aprendizaje puesto que el autor enfatiza que la construcción del conocimiento implica aspectos pedagógicos, interpersonales y organizativos por parte del docente. La presencia del docente debe relacionarse con el desarrollo cognitivo y con un entorno positivo de aprendizaje, además de contemplar los contenidos, la cognición y contexto como partes integrantes del todo.

La literatura menciona un sin número de características, competencias y funciones que debe poseer el docente del siglo XXI, dentro de las cuales se pueden destacar que debe ser flexible, motivador, innovador en el proceso de enseñanza-aprendizaje, debe crear un ambiente agradable, debe conocer los medios pedagógicos actualizados, debe orientar a los educandos en las dificultades que se presenten, debe escuchar las opiniones de los educando y tomar en cuenta sus aportes.

Para lograr estas competencias hay que cambiar el paradigma "Yo enseño", "El alumno aprende" ya que las estructuras de las universidades son del siglo XIX, los profesores siguen actuando como en el siglo XX y los estudiantes están en el siglo XXI. Por lo tanto, el perfil que el docente requiere para transformar el proceso de enseñanza-aprendizaje es el de Educar-Formar para la vida en las dimensiones: profesional, social y personal (De Armas, 2019). Este planteamiento nuevamente 
remarca la ineludible importancia de concebir la educación para el siglo XXI desde una perspectiva holística del aprendizaje.

Aparte de lo mencionado anteriormente, es de mucha relevancia exponer la visión de Tedesco (2010) el cual plantea que la educación del siglo XXI se apoya en dos grandes pilares: aprender a aprender y aprender a vivir juntos. El aprender a aprender plantea que la educación ya no podrá estar dirigida a la transmisión de conocimientos y de información sino a desarrollar la capacidad de producirlos y de utilizarlos. La función del docente consiste desde este punto de vista, en la tarea de enseñar el oficio de aprender, en la cual el oficio del educando está basado en una dosis muy alta de instrumentalismo, dirigido a obtener los mejores resultados posibles de acuerdo a los criterios de evaluación. El proceso de aprender a aprender es la manera cómo los educandos encuentran, retienen, comprenden y operan sobre el saber en el proceso de resolución de un determinado problema.

Entonces, el papel del docente es la de un acompañante cognitivo, donde muestra cómo se hacen las cosas. Es decir que se enfatiza en la práctica y en lo explícito. Este enfoque requiere de un esfuerzo mucho mayor en el proceso de aprendizaje tanto del docente como de los educandos. Por último el autor refiere que aprender a aprender también modifica la estructura institucional de los sistemas educativos en la que ya no es posible concebir la educación como una etapa de la vida sino aceptar que aprendemos a lo largo de nuestro ciclo vital.

Por otra parte, el aprender a vivir juntos se traduce en promover un valor tan esencial como es la solidaridad lo que permite que la sociedad tenga un buen nivel de desarrollo. "Vivir juntos, en cambio, siempre ha implicado la existencia de un compromiso con el otro" (Tedesco, 2010, p.38). Si se logra reflejar esto en los contextos educativos permitirá formar personas respetuosas, solidarias, tolerantes y que logren resolver los conflictos a través del diálogo.

En resumen, debemos tomar en consideración estos dos importantes pilares en nuestro rol docente, de manera que en al aula de clase y en cualquier contexto educativo se refleje claramente el aprender a aprender y aprender a vivir juntos.

\section{Rol de los contenidos}

La perspectiva holística del aprendizaje requiere que se cambien los contenidos de lo que se enseña y se aprende en la escuela o en la universidad. Delval (2013) hace referencia a que "los contenidos escolares deberían tener como objeto primordial la vida en su conjunto, y se debería de tratar de todo lo que afecta a los individuos” (p.10).

Delval (2013) hace mención que al enseñar se debe partir de las necesidades y de los intereses de los educandos y nuevamente sale a relucir la motivación puesto que 
el autor destaca que tenemos que fomentar la pasión por conocer, la curiosidad, que todos los individuos manifestamos en algún momento de nuestras vidas. La escuela o la universidad en lugar de apagar esa pasión deben hacer esfuerzos por reactivarla.

Esto se puede lograr si los contenidos que se abordan se refieren a problemas que afectan a las personas, los problemas cotidianos de la vida, de las relaciones humanas, de la televisión, del deporte, de la vida política y social. En fin contextualizar los contenidos.

En este sentido, el autor señala que lo importante de los contenidos es que nos permitan saber usar el conocimiento, saber buscarlos, darles un sentido para después aplicarlos. Entonces, hay que transmitir la idea de que la ciencia y el conocimiento sirven para resolver problemas, para mejorar la vida y para encontrarle un sentido.

En concreto, en la escuela para el siglo XXI no se deben promover los contenidos memorísticos, se debe reemplazar de la forma más inmediata el aprendizaje memorístico por el aprendizaje significativo en donde el educando descubra la relación entre el contenido y la realidad, la práctica, la aplicabilidad a su contexto individual, social y cultural.

\section{Conclusiones y perspectivas futuras}

La educación para el siglo XXI debe abordarse desde una perspectiva holística del aprendizaje puesto que la educación es un proceso humano complejo en el que es importante tomar en cuenta las características biológicas, cognitivas, psicológicas, afectivas-emocionales y culturales del individuo.

El proceso de enseñanza-aprendizaje por lo tanto también debe analizarse desde una visión holística, donde se enfatice en la individualidad del educando y del docente. Y se tenga claro que en los procesos educativos intervienen diversos factores institucionales, pedagógicos, sociodemográficos y psicosociales que determinan el éxito o fracaso académico de los educandos. Es fundamental profundizar en la identificación de estos factores pero es aún más relevante buscar posibles soluciones y no continuar evitándolos.

El educando desde esta perspectiva holística del aprendizaje es un sujeto activo, que construye el conocimiento desde su experiencia y la información que recibe. Además el educando debe comprender que la educación es un fenómeno dinámico por lo tanto él también debe poseer ese dinamismo puesto que se desenvuelve en un mundo contemporáneo donde los cambios se producen con mucha rapidez.

El docente por su parte, debe asimilar que el educando es un ser humano biológico, psicológico, social y cultural y que esas dimensionen influyen en el proceso de 
enseñanza-aprendizaje por lo que es preciso plantear estrategias que se adecuen a las características individuales de los educandos. En este aspecto, es necesario resaltar que en el aula de clase ya no debe existir la centralidad del docente, la linealidad y jerarquía sino más bien la promoción de relaciones de horizontalidad y no linealidad.

Desde este punto de vista, el docente desempeña un rol de facilitador cuya tarea principal debe apoyarse en dos grandes pilares: aprender a aprender y aprender a vivir juntos.

Por último, la perspectiva holística del aprendizaje requiere que en la educación para el siglo XXI se cambien los contenidos de lo que se enseña y se aprende. Los contenidos deben ser asequibles para el educando y no deben utilizarse para lograr un aprendizaje memorístico sino para promover un aprendizaje significativo en el que apliquen esos contenidos a situaciones prácticas de la vida real; en contextos actuales y cotidianos; para la solución de problemas, sólo de esta manera el educando hallará sentido a lo que está aprendiendo.

En la UNAN-MANAGUA hemos dado importantes pasos para los cambios que se requieren para la educación del siglo XXI, sin embargo cada día debemos mostrar compromiso para el mejoramiento de la calidad de los procesos educativos, avanzar y no detenernos porque la sociedad actual a la que nos debemos como una Institución de Educación Superior Pública así lo requiere.

\section{Agradecimiento}

Agradezco al Doctor Julio César Orozco Alvarado por el apoyo brindando para la realización del presente ensayo.

\section{Lista de referencias}

Cabrera, J. (2013). Los estilos de aprendizaje en el marco de una Educación Superior más humanista y desarrolladora. Revista Científica Estudios e Investigaciones, 2(1), 159-192. Recuperado de http://revista.unibe.edu.py/index.php/rcei/ article/view/110

Cabrera, J. (2009). La comprensión del aprendizaje desde la perspectiva de los estilos de aprendizaje. Recuperado de https://ebookcentral.proquest.com/lib/bibliotecaunansp/reader.action?docID $=3183072$

Chadwick, C. B. (2006). La psicología de aprendizaje del enfoque constructivista. Recuperado de https://ebookcentral.proquest.com/lib/bibliotecaunansp/reader.action? docID $=3170029$ 
De Armas, R. (21 de mayo 2019). Docencia, innovación curricular y competencias: El rol del profesor universitario. Conferencia llevada a cabo en Managua: Universidad Nacional Autónoma de Nicaragua.

Delval, J. (2013). La escuela para el siglo XXI. Sinéctica, 40, 1-18. Recuperado de http:// www.redalyc.org/pdf/998/99827467002.pdf

Espinoza, E. (2017). El aprendizaje en estudiantes universitarios. Recuperado de https:// ebookcentral.proquest.com/lib/bibliotecaunansp/reader.action?docID $=5214292$

Fernández, L. (1993). Educación y personalidad ¿posible estandarización?. Educación y Ciencia, 2 (7), 47-50. Recuperado de http://educacionyciencia.org/index.php/ educacionyciencia/article/view/58

León, A. (2007). Qué es la educación. Educere, 11(39), 595-604. Recuperado de https:// www.redalyc.org/pdf/356/35603903.pdf

Maldonado, C. E. (2014). ¿Qué es eso de pedagogía y educación en complejidad?. Intersticios sociales, (7), 1-23. Recuperado de http://www.scielo.org.mx/ scielo.php?script=sci_arttext\&pid=S2007-49642014000100002\&lng=es\&tln $\mathrm{g}=\mathrm{es}$

Santos Rego, M. (2000). El pensamiento complejo y la pedagogía. Bases para una teoría holística de la educación. Estudios Pedagógicos, (26), 133-148. Recuperado de http://www.redalyc.org/comocitar.oa?id=173514139012

Silva, J. (2010). El rol del tutor en los entonos virtuales de aprendizaje. Innovación Educativa, 10(52), 13-23 Recuperado de https://www.redalyc.org/ pdf/1794/179420763002.pdf

Tedesco, J. C. (2010). Desafíos de la educación en el siglo XXI. Revista Iberoamericana de Educación. 55 (2011), 31-47. Recuperado de https://rieoei.org/RIE/article/ view/524 